\title{
A case of peritoneal encapsulation syndrome
}

\section{T L A Janath Kumara ${ }^{1}$ and S K Kollure ${ }^{1}$}

\section{Introduction}

Peritoneal encapsulation [PE] is a rare condition characterised by a peritoneal sac covering the small intestine. This was first described in 1868 by Cleland [1]. Most patients with PE develop acute or sub acute small intestine obstruction. We report a patient with asymptomatic PE.

\section{Case report}

A 44-year old woman presented with rapid weight gain, generalised weakness and nocturia of 6 months. On examination she had trunkal obesity, a buffalo hump, and hypertension. Abdominal examination was unremarkable except for striae. A clinical diagnosis of Cushing's syndrome made and was confirmed by abnormally high serum cortisol. A CT scan showed a right adrenal tumour.

${ }^{1}$ General Surgical Unit, National Hospital of Sri Lanka, Colombo.

Correspondence: TLAJK, e-mail: <janathliyan@yahoo.com>. Received 2 June 2008 and revised version accepted 1 January 2009. Competing interests: none declared. 
At operation the small intestine was covered in a sac made up of thin peritoneal membrane from duodenum to terminal ileum. The membrane was found inferior to the omentum and transverse colon. It was attached laterally to the parietal peritoneum, medially to the ascending and descending colon, superiorly to the base of the transverse mesocolon and caudally to the posterior parietal peritoneum. The small intestine was visible through the membrane and appeared normal. The right adrenal tumour which was macroscopically malignant was excised. Peritoneal covering of the small intestine was left undisturbed. She made an uneventful recovery. Histology of the tumour revealed a well differentiated adreno-cortical carcinoma.

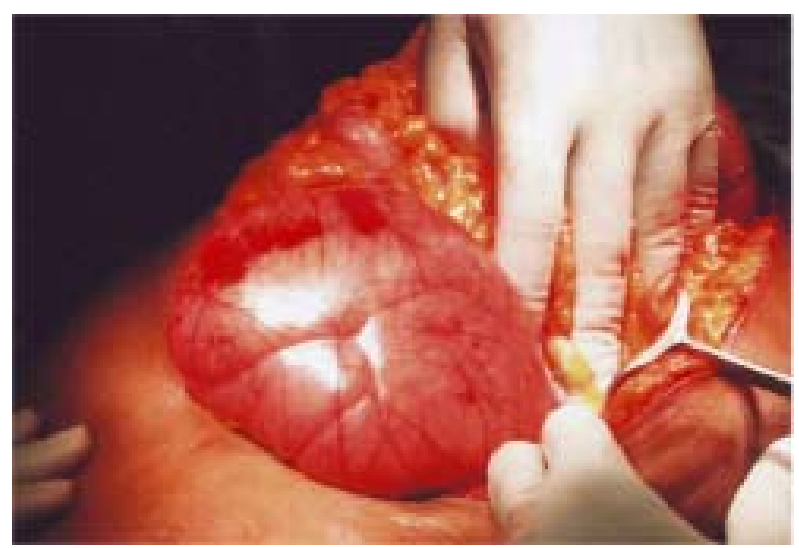

Figure 1.

\section{Discussion}

A membranous covering over the small intestine is a rare condition presenting almost always as intestinal obstruction. This condition is interchangeably described as peritoneal encapsulation, abdominal cocoon or sclerosing encapsulated peritonitis [2,3].

It is a congenital abnormality characterised by the small intestine lying behind an accessory peritoneal membrane. It is believed to be due to abnormal return of the mid gut loop to the abdominal cavity in the early stages of development. The small intestine is thus covered by a peritoneal sac derived from the original dorsal mesentery or from the yolk sac. Patients are usually asymptomatic but may present as acute intestinal obstruction. Rarely, like in our patient, the condition may be detected as an incidental finding. Patients with PE may not show characteristic radiological features if the membrane is very thin as in this patient. We did not disturb the peritoneal covering during surgery because of the risk of secondary adhesion formation and poor prognosis of the large malignant adrenal tumour.

Unlike this congenital form, the acquired disease (also called abdominal cocoon, or sclerosing encapsulated peritonitis) will have a thick greyish white membrane [2]. It usually presents with intestinal obstruction and has a mortality of $50 \%[2,4]$. This condition is described in association with chronic ambulatory peritoneal dialysis, patients on long term practalol (beta blocker), tuberculous peritonitis, sarcoidosis, ventriculo-peritoneal and peritoneo-venous shunts and retrograde menstruation $[2,4,5]$.

\section{References}

1. Foo KT, Ng KC, Rauff A, Foong WC, Sinniah R. Unusual small intestinal obstruction in adolescent girls: the abdominal cocoon. British Journal of Surgery 1978; 65: 427-30.

2. Eltringham WK, Espiner HJ, Windsor CW, Griffiths DA, Davies JD, et al. Sclerosing peritonitis due to practolol: a report on 9 cases and their surgical management. British Journal of Surgery 1977; 64: 229-35.

3. Ping Xu, Li-Hua Chen, You-Ming Li. Idiopathic sclerosing encapsulating peritonitis (or abdominal cocoon): a report of 5 cases. World Journal of Gastroenterology 2007; 13: 3649-51.

4. Lalloo S, Krishna D, Maharajah J. Abdominal cocoon associated with tuberculous pelvic inflammatory disease. The British Journal of Radiology 2002, 174-6.

5. Santos VM, Barbosa Junior ER, Lima SHM, Porto AS. Abdominal cocoon associated with endometriosis. Singapore Medical Journal 2007; 48: 240-2. 